\title{
Deutschsprachige Version des RadLex offiziell übergeben
}

Auf dem Jahrestreffen der RSNA vom 26. November bis 1. Dezember 2017 in Chicago übergaben Vertreter der DRG die deutschsprachige Version des RadLex an ausgewählte Funktionsträger der RSNA. 45000 radiologische Begriffe haben die DRG-Arbeitsgemeinschaften und die assoziierten Gesellschaften in zweijähriger Arbeit übersetzt, ergänzt und überprüft. Ein Mammutprojekt, dessen erfolgreicher Abschluss beweist, wie hervorragend die deutsche Radiologie aufgestellt ist und zusammenarbeitet. Prof. Dr. Thomas Hackländer, Vorsitzender der AG Informationstechnologie, lässt im Interview das Projekt Revue passieren und erläutert, wie RadLex den radiologischen Arbeitsalltag in Deutschland bereichern kann.

\section{Herr Professor Hackländer, sind Sie zufrie- den mit dem nun abgeschlossenen Pro- jekt einer deutschsprachigen Version des RadLex?}

Ja, auf jeden Fall! Durch die gemeinsame Anstrengung aller Arbeitsgemeinschaften der DRG ist es gelungen, eine qualitativ hochwertige Übersetzung des RadLex in dem gesteckten Zeitrahmen an die RSNA zu übergeben.

\section{Wie kam es zu der Idee, das RadLex ins Deutsche zu übersetzen?}

Die Idee zu einer deutschen Übersetzung des englischsprachigen RadLex wurde beim „KIS-RIS-PACS und DICOM-Treffen“ im Juni 2015 geboren, wobei der Bedarf insbesondere von der Industrie formuliert wurde. Um die fachliche Kontrolle über diese Begrifflichkeit nicht in die Hände von Industrieunternehmen, sondern in die der entsprechenden Fachgesellschaft zu legen, hat sich der Vorstand der DRG rasch überzeugen lassen, das Projekt zu unterstützen. Über einen Zeitraum von neun Monaten konnten in intensiven Gesprächen mit der RSNA die rechtlichen Rahmenbedingungen soweit geklärt werden, dass im März 2016 ein „Memorandum of Understanding“ zwischen RSNA und DRG unterzeichnet werden konnte. In den darauf folgenden sechs Monaten wurden unter Federführung eines kleinen Kernteams der AG Informationstechnologie die technischen Einzelheiten des Datenaustausches zwischen RSNA, DRG und Experten geklärt, sowie eine Guideline für die Bearbeitung erstellt. Die verbliebene Zeit von etwas mehr als einem Jahr bis zur Übergabe beim Jahrestreffen der RSNA in Chicago wurde je zur Hälfte für eine professionelle Übersetzung und die Überprüfung durch die Experten der DRG genutzt.

Die Übersetzung erfolgte ja in zwei Schritten - eine erste Vorlage lieferte ein eigens hierfür beauftragtes Übersetzungsbüro, die dann von den Arbeitsgemeinschaften geprüft wurde. Hat sich diese Vorgehensweise bewährt?

Dieses Vorgehen war sicher sowohl unter Zeit- als auch Qualitätsgesichtspunkten der richtige Weg. Den ehrenamtlichen Experten innerhalb der AGs wäre es nicht zuzumuten gewesen, die Fülle von Konzepten in einer vertretbaren Zeit in allgemein akzeptierte deutsche Begriffe zu übersetzen. Um eine möglichst hohe Qualität zu erzielen, haben wir in einer Ausschreibung eine Probeübersetzung von 100 zufällig ausgewählten Konzepten von den Übersetzungsbüros angefordert und schließlich den Anbieter ausgewählt, der das beste Verhältnis von Qualität zu Kosten angeboten hatte. Mit diesem Büro konnten wir auch vereinbaren, dass nur ein Übersetzer für unser Projekt zuständig war, sodass eine homogene Übersetzung entstanden ist. Die Experten der Arbeitsgemeinschaften konnten sich dann darauf beschränken, die schon hochwertige Übersetzung zu kontrollieren bzw. zusätzliche Synonyme

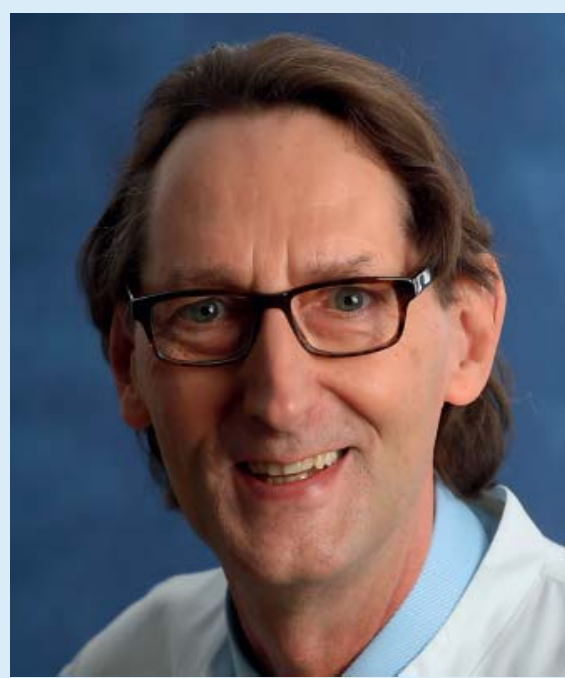

Prof. Dr. Thomas Hackländer

und Abkürzungen für die Konzepte zu ergänzen.

Wie hoch war der tatsächliche Bearbeitungsaufwand seitens der Arbeitsgemeinschaften?

Der Bearbeitungsaufwand zur fachlichen Überprüfung der Übersetzung durch die Experten der verschiedenen AGs war erheblich: Das RadLex umfasst mehr als 45000 Konzepte, die auf 14 AGs verteilt wurden, wobei deren Anzahl pro AG entsprechend der fachlichen Zuordnung zwischen 1600 und 7800 variierte. Zusätzlich haben sich die assoziierten Fachgesellschaften DeGIR und DGNR an der fachlichen Überprüfung beteiligt. Hier ist insbesondere das Engagement der DGNR hervorzuheben, die mit über 15000 Konzepten etwa ein Drittel der Arbeit geleistet hat. Den an der Überprüfung beteiligten etwa 40 Experten sei an dieser Stelle ausdrücklich gedankt. Ohne ihr umfangreiches Engagement wäre das Projekt nicht zu realisieren gewesen. 
Die deutschsprachige Version des RadLex wurde im Dezember 2017 in Chicago an die RSNA übergeben. Was passierte da genau bzw. in welcher Form und an wen wurde die Übersetzung weitergereicht?

Die Übergabe fand im Rahmen eines Arbeitsessens statt, an dem 14 Funktionsträger der RSNA und der DRG teilnahmen. Die formale Übergabe erfolgte durch den Präsidenten der DRG, Herrn Prof. Stefan O. Schönberg, an den Direktor für Informatik der RSNA, Dr. Christopher Carr. Nach einer Projektpräsentation durch den Vertreter der AG Informationstechnologie schloss sich eine Diskussion über die Erfahrungen und die zukünftige Zusammenarbeit an. Die RSNA hatte zusätzlich einen Vertreter der brasilianischen und der europäischen Röntgengesellschaft zu der Veranstaltung eingeladen. Beide informierten sich über die Erfahrungen in Deutschland, da es bereits konkrete Pläne für eine portugiesische Übersetzung und Ideen zu Übersetzungen in andere europäische Sprachen gibt.

Wie ist die weitere Pflege des Datenbestands organisiert?

Der Vertrag mit der RSNA sieht eine Pflege des Datenbestandes vor, die auf deutscher
Seite über das Kernteam in der AG Informationstechnologie koordiniert wird. Dabei sind drei Aktualisierungswege zu berücksichtigen: Korrekturen an der bereits bestehenden deutschen Übersetzung, Übersetzung neuer Konzepte, die von Seiten der RSNA eingebracht werden und Aufnahme neuer Konzepte auf Vorschlag von Anwendern in Deutschland. Um diese Aufgaben auch langfristig wahrnehmen zu können, wird eine spezielle Website auf der DRG-Homepage eingerichtet werden, über die alle interessierten Kreise ihre Wünsche einbringen können.

Welche konkreten Anwendungsfelder sehen Sie für das RadLex unmittelbar und welche unterstützenden Maßnahmen sind für seine Implementierung in den radiologischen Arbeitsalltag geplant?

Die Übersetzung des RadLex ist innerhalb der DRG in eine Befundungsinitiative integriert. Darin geht es unter anderem um die Entwicklung von strukturierten Befundvorlagen. Durch eine zusätzliche Kodierung der Inhalte mit Begriffen aus dem RadLex wird eine computergestützte Auswertung der Inhalte erleichtert. Ein anderes Projekt der AG Informationstechnologie beschäftigt sich mit der Kodierung aller Prozeduren in der Radiologie von der Leistungsanforde- rung bis hin zur Leistungsabrechnung. Hierzu gibt es einen internationalen Ansatz, der wesentlich auf der RadLex-Kodierung von Anatomie und Technik basiert. Darüber hinaus können wir über das RadLex zukünftig innovative Anwendungen erwarten: Anders als andere Kodierschemata wie LOINC oder SNOMED handelt es sich bei RadLex um eine Ontologie: Zu einem Konzept gehört nicht nur der deutsche und englische Begriff und ein Code, sondern es ist auch die Abhängigkeit des Konzeptes zu anderen Konzepten hinterlegt. So kann man aus dem Code für ein Astrozytom IV implizit ableiten, dass es sich um eine bösartige Neubildung des Gehirns handelt. Dieses Wissen kann Systemen mit künstlicher Intelligenz helfen, Inhalte besser zu verstehen und Schlussfolgerungen zu ziehen.

Ist der Zugang zum deutschsprachigen RadLex kostenpflichtig?

Der mit der RSNA geschlossene Vertrag sieht vor, dass die deutsche Übersetzung des RadLex - genau wie der Originaltext kostenfrei in allen Anwendungen genutzt werden darf. Änderungen der deutschen Übersetzung eines Konzeptes dürfen aber nur von der DRG vorgenommen werden. 